Complejidades en la investigación de la transformación del saber artístico en saber \title{
escolar
}

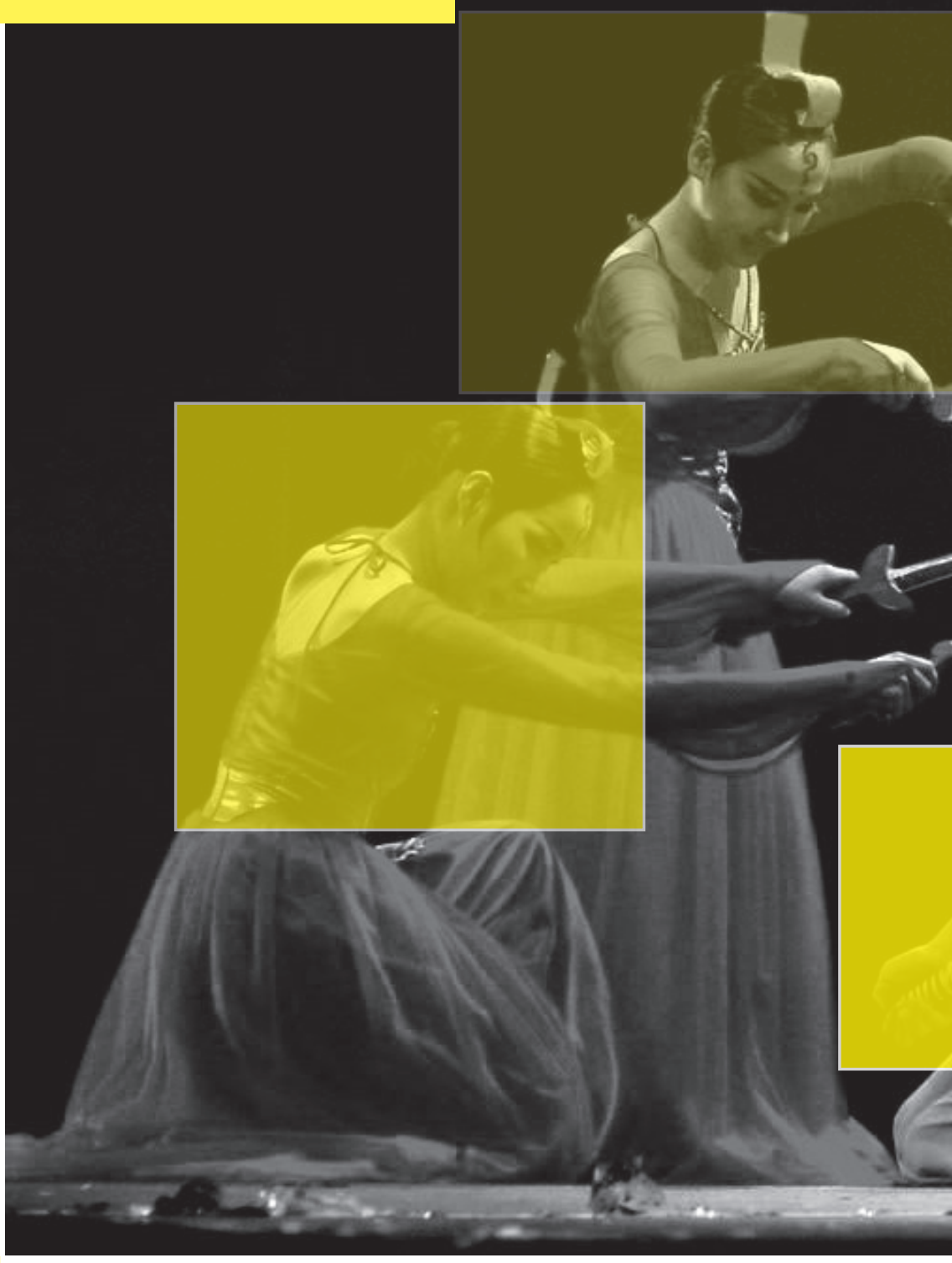


con efectos directos sobre los saberes, sobre la visión que de éstos se ha tenido (recuérdese que el concepto mismo de ciencia ha hecho crisis ${ }^{1}$, como el de arte en sus cánones), sus discursos y dispositivos.

En la ruta de demarcar la transformación de saberes para la escuela es ineludible tener en cuenta estos elementos junto con: a) el destinatario de los saberes (escuela primaria, secundaria, etc.); b) quién determina qué enseñar y c) el profesor, su ejercicio y los contenidos que despliega.

Así las cosas se niega la disyunción en y entre los saberes y se afirma, al menos provisionalmente que hay otros saberes que se deben incluir en el estudio de la transformación de los mismos en escolares y que para su desciframiento es menester estudiar tanto los conocimientos del profesor como lo que hace en el aula.

En nuestro país aún hacen carrera, -para citar el ejemplo del teatro-, presunciones como que para enseñar basta con conocer la disciplina, que se aprende haciendo o por imitación, que la investigación y la docencia

\footnotetext{
La noción misma de ciencia se interpela en los márgenes de la antropología, la sociología y la sociología del conocimiento o del pensamiento complejo. Basta disfrutar de posturas como las de Hugo Zemelman quien se interroga por los límites de la ciencia a partir de una nueva postura sobre el sujeto, la realidad y cómo ella se piense, la de Jesús Ibáñez desde los paradigmas de segundo orden o la de Yehuda Elkana (1977) quien emparenta la ciencia con sistemas culturales como el arte, los mitos o la religión.
}

teatral sólo puede estar en manos de "especialistas teatrales”, que la investigación misma hace parte de todo proceso creativo, de puestas en escena o de experimentaciones con miras a la búsqueda de nuevos itinerarios estéticos. Perspectiva que ejemplifica la (sobre) valoración del saber artístico y refleja qué temas pedagógicos son asunto de otros especialistas cuyos dispositivos se adicionan sin adelantar discusiones sobre nuevas visiones del arte en la escuela o los aspectos de orden didáctico de las disciplinas.

Esta endogenización ha mellado aspectos del teatro mismo (quizá de las artes) y del lugar que estas debieran ocupar en la escuela. El arte, en tanto práctica social, merece una proyección vigorosa y un posicionamiento teórico para entrar en diálogo con la educación, la sociedad, con otras disciplinas y por supuesto, con la vida y la naturaleza. En una ciudad como Bogotá, en la cual circula un gran volumen de productos teatrales, con universidades que ofrecen programas de formación en las artes -en este encuentro nos informan que son 44 ¡sólo en Bogotá!-, con la proporción de festivales y de apoyos provenientes de entidades públicas, asombra la proscripción de referentes teóricos, de la reflexión sistemática sobre la educación artística, sus avatares, sus retos en la sociedad de la información y del conocimiento, su lugar en el contexto de la globalización y casi todos los aspectos relacionados con la formación, las experiencias existenciales y las estéticas y la espiritualidad.

Aún es notoria la ausencia de sistematizaciones e investigaciones por parte de los agentes artísticos, culturales y educativos, situación que se suma al incremento de procesos de formación no formales (cuya caracterización está por hacerse), el hecho de que los programas universitarios adolecen de espacios de encuentro, debate y proposición $\mathrm{y}$, que el arte ha tomado formas estéticas y escolares que aún no se descifran.

Cabe en este contexto ubicar discusiones sobre aspectos didácticos de la educación artística, los cuales a mi parecer aglutinan los restantes y permiten dar cuenta de las singularidades de la formación artística, como de los contenidos, los programas curriculares, los procesos de aula, el conocimiento del profesor, sus creencias, experiencias, concepciones y particularmente, lo que hace el profesor en la clase.

Apropósito de la formación, sabido es que la mayoría de los procesos de formación teatral del país descansan en la empiria, el activismo o la imitación, ello no es óbice para el desarrollo del tema en cuestión. Se debe en gran parte a estas experiencias, tanto la emergencia del teatro en el país como la creación de escuelas de formación. No obstante, el mercado potencial ha crecido, el número de escuelas y de programas formales y formalizados. ¿Cuáles son los paradigmas de formación?, ¿qué premisas los sustentan? ¿En qué marcos de referencia se sitúan los programas de formación de educadores? ¿Qué peculiaridades le diferencian de un proyecto de formación de actores? Qué concepciones de formación, escuela, arte (teatro) y saber didáctico circulan?

Uno de los grandes maestros teatrales (amén de reconocido director, teórico, y dramaturgo) se refería al aprendizaje teatral desde el ángulo de la "transmisión de la herencia"; para Barba, a la pregunta acerca de cómo se forma un actor, respondía enfatizando la presencia de los maestros quienes ayudan a descubrir el propio camino; de un "tótem” (un artista a emular) y del proceso orgánico (lo que se es y lo que se sabe son la base de este proceso) (Barba, 2003: 257) ${ }^{2}$. Si bien el actor, su cuerpo, su espíritu y su mente imbricados, constituyen la materia orgánica del proceso de formación, ésta no es posible sin unos métodos, sin unas técnicas que facilitan la mayor eficacia de la acción, sin unas prácticas, sin unos gestos, medios, situaciones y contratos didácticos como diría el maestro Rickenmann.

Los aspectos didácticos del teatro, -sigo con la ejemplificación-, se reducen a esquemas lúdicos, a formas como los sociodramas, psicodramas, juego expresivo o dramático, etc. Nótese el lugar periférico que siguen ocupando las artes, la carencia de libros de texto escolar en artes y el hecho de que muchos profesores, al menos en teatro, tienen títulos en áreas distintas. Diríamos que asumir el estatuto de saber artístico en la escuela es posible gracias al estudio de lo que hace el profesor de artes, a los objetos culturales que despliega en la escuela, a los dispositivos discursivos y no discursivos, a sus ingenierías didácticas.

\section{En la investigación de la}

\footnotetext{
A pesar de que la publicación reseñada corresponde al año 2003, el texto con ese título fue publicado en 2000 bajo el título "Tacit Knowledge: heritage and waste”, en la revista New Theatre Quaterly. № 3.
} 
transformación: el valor del profesor, de su saber y la potencia de su actividad.

En la dinámica de la trasposición de los saberes artísticos a la escuela tienen su lugar dos aspectos, por un lado, se advierte que el profesor debe estar en posesión de algo mucho más que un conocimiento disciplinar como se enfatizaba en el siglo XIX o pedagógico, como se acostumbra desde la centuria anterior. En este contexto aparece el concepto de profesionalidad del profesor que conduce, de nuevo, a lo que sabe, lo que hace y lo que es el profesor. En otras palabras, lo que define el conocimiento del profesor no sólo es el contenido (pedagógico, disciplinar, escolar, actitudinal, axiológico), ni el método, ni la rigurosidad, ni los esquemas de creencias, sino el conjunto de estos elementos articulados alrededor del saber experiencial, que es otro saber, efecto de la actividad, saber que emerge de la práctica -en lo que hace el profesor- y la experiencia actualizada del docente.

El saber del profesor

Se admite entonces, la existencia de un tipo específico de saber que le es particular a la profesión docente y que es necesario caracterizar y esclarecer para comprender los obstáculos en su aplicación y las dinámicas de su transposición en la escuela. La investigación sobre el conocimiento profesional del profesor es relativamente reciente, surge en el contexto anglosajón al promediar los años 70 y se ha ido desarrollando a partir de trabajos en Francia, Estados Unidos y España especialmente, definido en un comienzo como "el conjunto de conocimientos que los enseñantes usan en su práctica pedagógica” incluye un tipo de conocimiento didáctico y teórico junto con el conocimiento de los errores de los estudiantes que coadyuvan en la toma de decisiones y la elaboración de juicios (Bromme, 1988:19), tenemos pues, la vinculación entre el saber y la actividad del profesor (como práctico reflexivo) a la que se suman las creencias, el saber experiencial (Porlán, 1998) y las concepciones del profesor tanto de su oficio como de la materia que enseña.

Porlán, al hacer referencia al conocimiento del profesor lo subdivide en uno profesional dominante y otro profesional deseable, éstos se enmarcan en una innovadora categoría de análisis llamada Hipótesis de Progresión mediante la cual plantea la existencia de dos niveles, uno inicial o mayoritario y uno de referencia o deseable (Porlán, 1998). Esta propuesta, promovida en múltiples trabajos del grupo de investigación, IRES ${ }^{3}$, se despliega a partir de la existencia de cuatro componentes (el conocimiento académico, las teorías implícitas, las creencias y experiencias y las rutinas) que se articulan mediante un saber metadisciplinar. Estos componentes se constituyen en las fuentes del conocimiento del profesor. La propuesta del grupo de Sevilla se destaca porque formula principios a tener en cuenta en la formación de profesores, ellos relacionados con contenidos formativos que emergen como consecuencia de integrar diversas epistemologías. Cabe tener en cuenta que Porlán insiste en que en la formación universitaria se forma con lo que él llama el modelo didáctico dominante, caracterizado por ser fraccionado (sin visión de conjunto, desintegrado); enciclopédico (se enseñan datos y definiciones); descontextualizado ("se ocultan condiciones científicas sociales, históricas e ideológicas en las que el conocimiento se construye") y absolutista (la ciencia como conocimiento verdadero) (Porlán, 2003:24). ¿Ocurrirá lo mismo en la formación del profesor de artes?

La complejidad del conocimiento del profesor exige la definición de categorías de análisis del mismo, en esa ruta Shulman (Shulman, 1986) reconoce las siguientes: Content Knowledge: Se refiere a la cantidad y organización del conocimiento per se en la mente del profesor. Pedagogical Content Knowledge: en esta categoría aborda el conocimiento del contenido pero se centra en los elementos de su enseñabilidad, e incluye las formas de representar y formular las temáticas con el fin de que sean comprensibles por otros, así como aquello que facilita o dificulta la comprensión de las temáticas. Curricular Knowledge: Se refiere al conocimiento sobre los programas diseñados para la enseñanza de una temática y sobre su conveniencia de adaptación y pertinencia a algunas circunstancias. Forms of Knowledge: formas de conocimiento aplicables a las tres categorías antes señaladas.

3 IRES: Grupo de investigación constituido en 1991 con este nombre, pero que desde los 80 trabajaba en Investigación en la Escuela pública; los primeros documentos constituyen la base del Proyecto Curricular "Investigación y Renovación Escolar (IRES). El grupo había surgido en la Escuela de Magisterio de la Universidad de Sevilla por iniciativa de Rafael Porlán y Pedro Cañal.
De esta manera, Shulman se interesa tanto por los contenidos como por su transferibilidad con el fin de que los estudiantes los aprendan (El CDC). Por ello la enseñanza es uno de los puntos nodales ya que en su realización se escenifican los saberes preconstruidos en el profesor en virtud de su formación, su sistema de creencias, rutinas y demás, en esta realización se elaboran una serie de constructos epistemológicos (los construidos por el profesor). Este otro saber es más global, integrador y sintético. La enseñanza está determinada no sólo por la especificidad de los contenidos a enseñar sino por los mecanismos propios para hacerlos comprensibles, estos mecanismos obedecen a un modelo didáctico personal que en la mayoría de los casos dificulta la evolución del conocimiento profesional del profesor. Muchos de los formadores piensan que haber aprendido postulados, teorías o técnicas habilita para enseñar, pero es necesario como dice Porlán construir un conocimiento genuino (Porlán y Rivero, 1998): el conocimiento del profesor devenido de la conjunción entre el saber académico, las rutinas, las creencias, las teorías implícitas (ideas, conceptos), las concepciones de enseñanza, el saber generado por la experiencia, etc.

Se reitera: el conocimiento del profesor emerge de los complejos procesos organizados alrededor de la enseñanza. En ella, por medio de ella, los saberes son reelaborados, integrados y transformados, proceso que a su vez produce sentidos, significados y teorías prácticas fundamentales. En este sentido la categoría CDC 
permite trascender los conocimientos estrictamente disciplinares, constituye, además, el prisma desde el cual identificar el saber del profesor construido en la práctica, a partir de sus concepciones, de los saberes académicos y experienciales. La práctica del profesor, sus “gestos” tienen unas dinámicas y una naturaleza propias, de allí la pertinencia de un ámbito epistemológico peculiar y concreto: la praxis, la práctica en tanto intervención, no acción elemental sino actividad intencional.

\section{En la enseñanza del arte emerge un saber cualitativamente emergente, diferenciado, sincrético y enmarcado en la experiencia y la actividad práctica}

La educación artística es -o debería ser- un campo problemático, se enseña una disciplina deformada, una práctica social vinculada a circunstancias histórico culturales, mutantes e híbridas (al menos en teatro son varios los objetos que entran en juego). No obstante, en la transposición de los objetos artísticos y culturales en la escuela se hace necesario considerar aspectos como los saberes experienciales, la actividad del profesor, sus creencias (saberes que posee pero que no son explícitos). Según Porlán los profesores trabajan con diferentes tipos de conocimiento, se pretende, en consecuencia, dar cuenta de un conocimiento emergente a partir de este sincretismo -que había permanecido en estado de subvaloración-, para así promover otros conocimientos exentos del dominio de los saberes disciplinares y académicos.
Así, la práctica educativa, lo que hace el profesor, se concibe como un saber, un saber hacer, que entraña a su vez una suerte de competencia, destreza y habilidad que le acercan a la inteligencia, la utilidad, la organización y la orientación. La práctica enseña y el profesor enseña por su práctica (Tardif, 2004: 111). La enseñanza es en sí misma una actividad práctica, intencional y pensada. Es una actividad donde el pensamiento y la palabra se repliegan sobre sí, donde se conjugan realidad y utopía, técnicas y raciocinios, normas y subversiones, hábitos y rupturas, tradiciones e innovaciones, es, a fin de cuentas, movilidad y en consecuencia creación, este asunto que pudiera pasar inadvertido o que tiñe con cierto pragmatismo la labor educativa es relevante en el oficio artístico y en el educativo. Para citar el caso del teatro y su enseñanza: esta no es posible sin la ejecución consciente de acciones pre-expresivas, expresivas y fisicovocales.

Lo anterior conduce a Dewey, para quien no es posible separar, en el seno de una experiencia vital, la práctica, la emoción y el intelecto, como tampoco, poner en evidencia las propiedades de uno de estos componentes dejando de lado las características de los otros dos, a enfatizar la importancia de la experiencia y la actividad práctica (en el sentido dispuesto por el mismo Dewey) es alejarse de exigencias endémicas que proliferan desde el siglo XIX y que se basan en el instrumentalismo, lo que ha impedido que se transforme una práctica (para el caso la práctica del profesor de artes) y la experiencia como objetos de conocimiento. 
El término «práctica» indica que el organismo dialoga con los eventos y los objetos que lo rodean. Desconocer que existe un conocimiento en la práctica del profesor es ocultar el valor de su trabajo, de su creatividad, de su saber hacer, de la posibilidad de instituir sentidos y significados a partir de su propia actividad. La aserción de Dewey nos recuerda que el sentido de los fenómenos, eventos y cosas se construye solamente a través de las actividades humanas; consecuentemente la práctica educativa no está desvinculada de los aspectos sociales que le constituyen, que el profesor tiene unos modos de funcionamiento original, un tipo de saber que tiene carácter social y cultural.

Los conocimientos prácticos configuran así un eje organizador de la profesión del docente, le facultan para enseñar. El conocimiento del profesor no sólo está limitado a los saberes disciplinares, científicos, artísticos o estéticos y educativos (los cuales no se pueden separar) sino a los producidos en la práctica educativa: el saber experiencial que a su vez demanda desarrollos conceptuales, circunscripciones epistemológicas, soportes investigativos. Esta idea encuentra argumentación en la noción de saberes pragmáticos, los saberes que "sirven de base a la enseñanza [y que] están ligados tanto al trabajo como a la persona del trabajador. Se trata de saberes ligados al propio cometido, de saberes sobre el trabajo, ligados a las funciones de los docentes. Mediante el cumplimiento de esas funciones, se movilizan, modelan, adquieren, así como demuestran también, las rutinas y la importancia que los educadores dan a la experiencia", (Tardif, 2004:77).

El enfoque aportado por Tardif contribuye en la cuestión del saber del profesor con la inclusión del trabajo y la acción en la enseñanza. Cabe soslayar que en la comprensión de ésta el autor señala como importante el hecho de que no se trata de una sola acción ligada a la enseñanza, "sino una diversidad de acciones heterogéneas. Los diferentes tipos de acción se encuentran en la práctica de los educadores profesionales” y que mediante la negociación y la interacción con otros actores propios del contexto socioeducativo producen un saber en la acción, "saber complejo que debe considerarse en función de los tipos de acción presentes en la práctica" (Tardif, 2004:130). No parece ahora tan obvio agregar que saber no es sinónimo de saber enseñar. No es sólo llevar a la práctica una teoría, es llevar a la práctica una práctica: una actividad educativa o didácticamente intencionada. Saber enseñar en la acción supone la integración de un conjunto de saberes.

Las actividades de formación en artes no están por fuera de la práctica. De una práctica especial, estructurada, formalizada, pre, co y posconstruida, actividad reflexionada en donde la técnica se vincula a una práctica formadora de otro tipo de conocimientos... a una práctica creadora y creativa. Y la creatividad tiene cabida (al menos la demanda de la sociedad lo expresa) en la configuración de nuevos modos de enseñar, de ser y de saber.

El análisis sobre la actividad del profesor como pivote de la investigación sobre la transformación de saberes en la escuela tiene incalculable valor en la corriente francesa, la cual -a partir de la teoría de la transposición didáctica (proceso por el cual el profesor reelabora el conocimiento 
de los científicos de manera que lo pueda proponer a los estudiantes sin simplificarlo, sin que se disipe el rigor y la teoría) y de la teoría de las situaciones de Brousseau-, se constituye como un intento por describir y teorizar los procesos didácticos a partir de la noción de terna didáctica (docente-saber-alumno). Una de las ventajas de esta perspectiva es la concepción sistémica de los procesos que son siempre analizados teniendo en cuenta los tres elementos de la terna y sus determinaciones mutuas; es decir, uno de los elementos se comprende desde los restantes.

La concepción del sistema didáctico se basa en la articulación de los elementos de la teoría histórico-cultural de la actividad (retomando a Léontiev y Vygotski especialmente). Ésta, en el contexto de la situación de enseñanza-aprendizaje, se asume como un proceso explicativo, como un sistema de organización de las prácticas humanas. En tal caso el sujeto es el motor de las actividades, pero éstas se configuran como el eje, el objeto de descripción, la unidad de análisis. La actividad es un acto entre el profesor y el estudiante, lo que en el argot de la clínica descriptiva se conoce como la 'construcción conjunta de la actividad de enseñanza-aprendizaje` (Sensevy y otros, 2007)

Para dar cumplimiento a una investigación sobre la transformación de saberes o contenidos artísticos en la escuela, es importante tener en cuenta que ha de efectuarse una pesquisa acerca de lo que hace el profesor (si acogemos que es escasa la investigación en asuntos relacionados con transposiciones artísticas en la escuela y, que lo que se despliega en ella es un enmarañamiento de saberes necesarios de elucidar), lo que incluye esclarecer asuntos como las metas de aprendizajes que se persiguen en artes, los objetivos de la dinámica enseñanzaaprendizaje, características de los enunciados y las consignas dentro del proceso de enseñanza, los saberes que se ponen en juego en situación de formación artística, estrategias, procedimientos y contenidos.

Con el fin de aclarar, invoco el teatro de nuevo, en donde existe una tradición en la formación actoral, la mayor parte de ella circunscrita en los métodos de maestros o directores (Meyerhold, Grotowski, Stanislavsky, Brecht, Barba, Michael Chejov, etc.) que cultivaron metódicamente una experiencia, la sistematizaron y la socializaron; ello, junto con la conformación de escuelas en las que se enseñaba tal o cual método, les dio validez y reconocimiento. Más adelante a las técnicas de formación del actor se fueron agregando complementos en las áreas de voz y cuerpo. Esta subespecialización incrementó el nivel de problematización en la formación, ya no se trataba de actuar, era imprescindible el dominio de otras prácticas, de otros recursos expresivos y en consecuencia la cualificación y presencia de especialistas.

Ahora bien, al hacernos la pregunta por lo que lo hace a uno profesor de teatro, se tendría, quizá un panorama más amplio: además de lo inmediatamente señalado, la escuela solicita del profesor otras destrezas que colindan con la dirección, la producción, la gestión y en algunos casos la dramaturgia, amén de la apelación al teatro en la constitución de nuevas maneras de relacionarse, en la resolución de conflictos o el desarrollo de competencias comunicativas y ciudadanas.
Vista así la función del profesor de teatro es más compleja de lo que comúnmente se cree y va más allá de un saber artístico o pedagógico. Es apenas obvio que la formación del profesor exige una serie de dominios entre los que se destacan saberes específicos disciplinares y pedagógicos, -más que su dominio particular la superación de la disyunción entre teoría y práctica-; un saber curricular (criterios para seleccionar, segmentar y programar contenidos), conocimiento del contexto y de estrategias para hacer frente a los múltiples problemas socioculturales, comportamentales, actitudinales y cognoscitivos de los alumnos, conocimientos de didáctica, gestión y evaluación; todos ellos relacionados con el carácter abierto, polifacético y dinámico de la educación artística, para responder así, creativamente ante la inmediatez (Gimeno Sacristán, 1993), la multiplicidad de frentes y tareas, en procura de flexibilizar lo aprendido para situarlo contextualmente y posicionarlo creativamente ante la complejidad, la indeterminación y lo incierto de la actividad pedagógica.

Pero allí no se agota el campo de discusión, desentrañar los aspectos que hacen posible la formación de un profesor para que transforme su saber artístico en escolar implica acercarse a preguntas no sólo acerca de lo que éste ha de saber, de los modos cómo construye su conocimiento, su función y relación en contextos específicos. Se ha de considerar su vivencia experiencial como artista, sus concepciones sobre arte, educación y el oficio de maestro, los cuales dadas las circunstancias del pensamiento en la contemporaneidad pueden tener estatuto de saber.

Precisando la dinámica de la investigación sobre la transformación se puede, por ejemplo, abordar los contenidos específicos de la formación de un actor o como se le conoce en el ámbito colombiano, de un maestro en arte dramático (contenidos de voz, cuerpo, actuación, poéticas teatrales, lectura, dirección, dramaturgia, historia del teatro, técnicas corporales de preparación, etc.) como el primer acercamiento al objeto de estudio para, en un "segundo momento” efectuar la reflexión didáctica, ello al estudiar los objetos del primer momento como objetos de enseñanza (Campo del teatro como conocimiento, campo del teatro como medio de desarrollo del niño, campo del teatro como medio de desarrollo de capacidades transversales en la escuela, etc).

En una torsión de los contenidos específicos del primer nivel se procuraría dar cuenta de su aplicación en el contexto de la enseñanza, de su transposición, de los cambios que los contenidos (y los saberes) sufren al ubicarse en la perspectiva de la aplicación en la escuela y de sus implicaciones 
y efectos curriculares. Lo anterior determinará una concepción del arte como saber que enseña y que se aprende para ser enseñado, una concepción del arte y la educación artística a distancia del arte como quedó “manifiesto” porque sabemos que el arte se aloja en la escuela pero aún no la habita.

Por ello abordar el sistema didáctico que se despliega en la educación artística, en las interacciones objetos-docente-discente, en los medios y en las situaciones, posibilitará una delimitación de los múltiples objetos presentes en la enseñanza que se transformarán o quizá sea suficiente con que ojalá empiecen a existir en las escuelas, en nosotros y en nuestras vidas.

\section{Bibliografía}

Barba, E. (2003). Obras Escogidas. La Habana, Ediciones Alarcos. Tomo I.

Bromme, R.(1988) Conocimientos profesionales de los profesores. En Revista Enseñanza de las ciencias. Vl. 6. № 1. Marzo. Barcelona.

Brousseau, G. (1986) Enseñanza y aprendizaje de la modelización. En Enseñanza de las ciencias. 4 (1), 45-50.

Chevallard, Y. (1991) La transposición didáctica. Del saber sabio al saber enseñado. Buenos Aires, Aique.

Gimeno Sacristán, J. (1993). Comprender y transformar la enseñanza.. Madrir. Morata.

Grossman, P. (1990) The making of a teacher: teacher knowledge and teacher education. New York. Teacher College Press.

Marcelo, C. (2006). La formación docente en la sociedad del conocimiento y la infor- mación. Ponencia presentada en el IV Encuentro Internacional KIPUS.

Marcelo, C. (Editor) (2001). La función docente. Madrid, Síntesis.

Porlán R. y Rivero A. (1998) El conocimiento de los profesores. Sevilla. Diada Editorial

Porlán, R. (2003). Principios para la formación del profesorado en secundaria. En Revista Interuniversitaria de formación del profesorado. Vol. 17 № 1. Zaragoza. Shulman. L. (2001) Conocimiento y Enseñanza. Ensayo. Estudios públicos.

Shulman L. "Those who understand: Growth in Teaching” En. Educational researcher Vol 15. No 2. 1986.

Tardif, M. (2004). Los saberes del docente y su desarrollo profesional. Madrid: .Narcea. Pg. 80,81 .

MIGUEL ALFONSO. Profesor de planta de la Universidad Pedagógica Nacional de donde se licenció en Español-Inglés, Psicólogo de la Universidad Nacional de Colombia, Especialista en Educación-Comunicación de la Universidad Central. Coautor de la Licenciatura en Artes Escénicas de la Universidad Pedagógica, programa que coordinó durante cerca de ocho años. Asesor de Instituto de Cultura y Turismo. Actualmente es estudiante del Doctorado interinstitucional en Educación de las universidades del Valle, Distrital y Pedagógica. mikealf8@yahoo.com
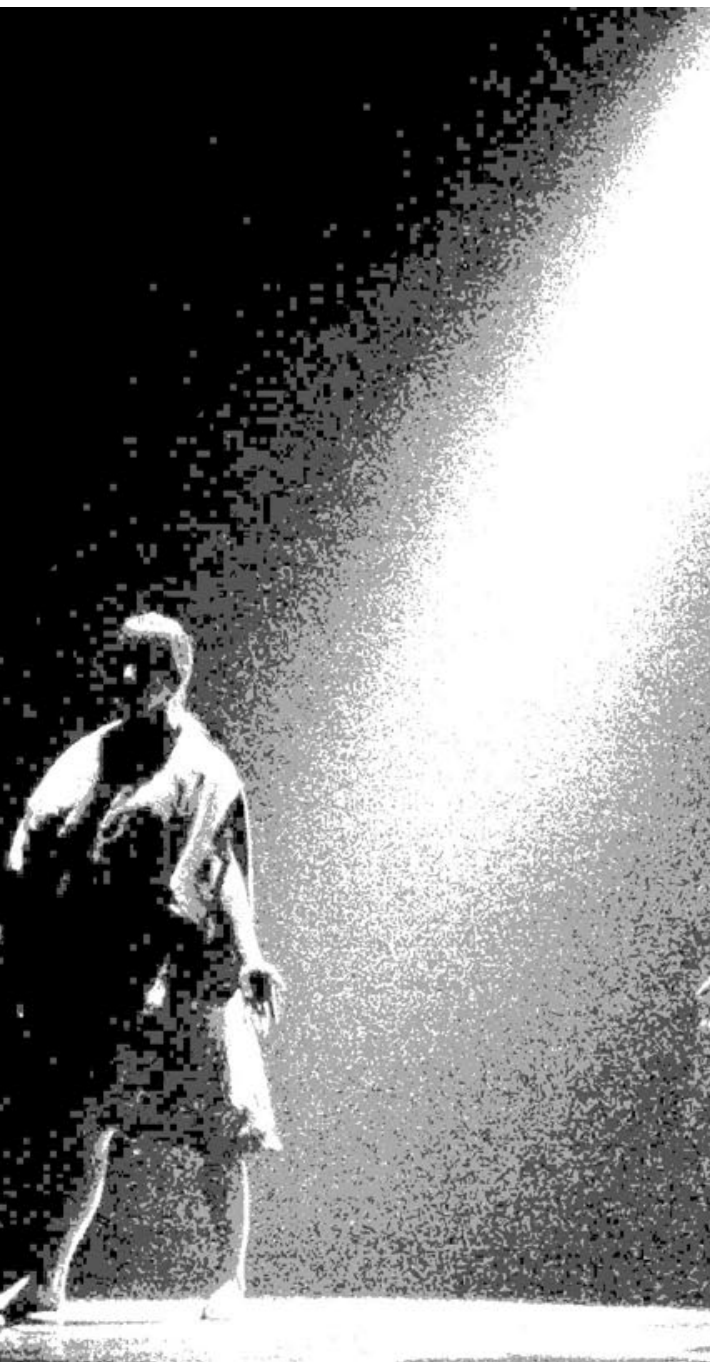
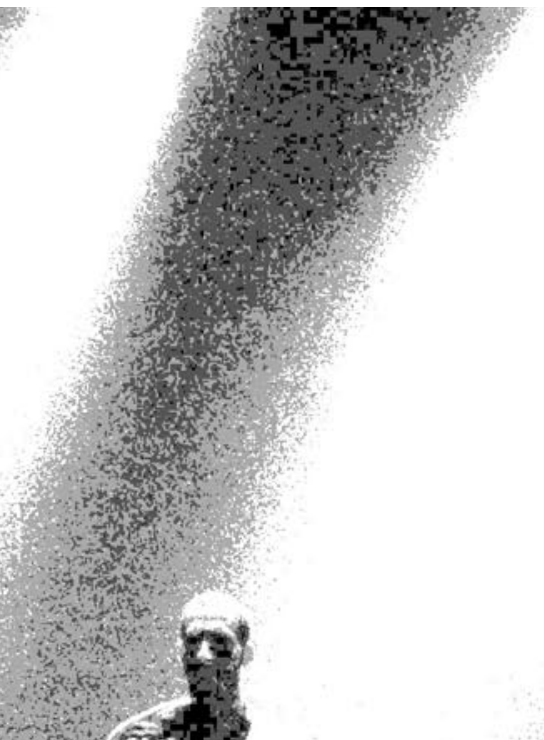
sing $+1$ 1. $+2$ 42 (1) 